\title{
EXTENSITY AND PITCH.
}

BY DR. KNIGHT DUNLAP,

University of Califormia.

Psychologists in general agree that 'sensation' has the four characters of quality, intensity, duration (or protensity), and feeling-tone. To these some would add volume (or extensity), and others would add vividness. There seems to be no reason why the list should not be somewhat further extended by adding local significance, which may with advantage be distinguished from quality; and meaning, or symbolic value, which is at least as important as any of the other characters. Even with these additions there is question whether the list is complete, but it is sufficiently so for present purposes.

These characters of simple sense objects are not parallel in their functions, but differ in their relation to the existence of these sense objects, and in their modes of variation. The first three which we have named seem to be essential; we can not conceive of the existence of a ' sensation' which has no duration, or which has no intensity, or which has no quality; $i . e .$, which is no particular kind of ' sensation.' Whether the same holds true for extensity is perhaps less certain, but there is strong evidence that way, as we shall see later. Vividness, feeling tone, local significance, and meaning, seem to be less essential. There conceivably may be sense objects present to consciousness which yet are of zero vividness; that is, they are either in the realm of so-called subconsciousness, or else they are neglected in their immediate nature, and their meaning alone taken into account. Local significance, again, may possibly be lacking; olfactory objects, in particular, never seem to have in themselves any local signs, although of course they are localized by the help of tactual and muscular experiences; something of the same kind seems to be true of auditory sensations also. It may be that local signs exist only in touch and sight; 
or on the other hand it may be that there is a system of local signs present in hearing which is represented by the system of pitches; but at least we can conceive of 'sensations' which should be entirely devoid of this character. Suppose, for instance, two nerve endings which should when stimulated produce sensual processes corresponding to the same sensuous quality; there might be in these processes nothing which would enable us to distingnish one from the other, and yet both would be real.

Sense objects may be neutral in feeling-tone, which is equivalent to their having no feeling tone. Some psychologists insist on calling neutrality a definite feeling-tone, thereby making feeling-tone an essential character; but this seems an uncalled for complication, since feeling-tone signifies the character by which states of consciousness differ as regards pleasantness (or unpleasantness, whichever you chose) and when this factor is reduced to zero there is no better excuse for saying that it still exists than there is for saying that zero intensity and zero duration are still existent intensity and duration.

Meaning, likewise, is not necessarily present. A sense object may conceivably be taken for just what it is, without reference to anything else. Of course, the more experience we have, the more nearly impossible this becomes; but even so we may hold that the absence of meaning does not imply the nonexistence of ' sensation.'

These various characters of simple sense objects show also diverse types of behavior in the analysis of psychical compounds. The mere quality of a compound involves nothing which is not in the qualities of its components. So the intensity and duration are direct functions of the intensities and durations of its elements. Or, if the more accurate form of statement is preferred, we may say that the qualities, intensities, and durations, of the elements into which the complex state is analyzed will include all qualities, intensities, and durations found in the complex, and no other; there is neither surplusage nor deficit resulting from the analysis. The complex as a whole has no quality which cannot be reduced to the qualities of the elements; no intensity which cannot be reduced to a summation of elementary intensities; and no duration which is different from the duration of a definite number of its elements. 
Extensity falls in with duration. But in both these characters we must be prepared for an apparent surplusage resulting from analysis, which apparent surplusage is due to the phenomena of superposition; $i$. e., simultaneity in time or collocation in space. When we take these factors into account the apparent post-analytical redundancy disappears.

With regard to the other characters of elementary sense objects, this simple analytic relation does not hold. There are factors in the feeling-tone of a complex that are not assignable to any of the sensuous elements into which the complex may be resolved, but which seem to belong to the complex as such, or perhaps to intellectual elements. The meaning of a complex is far different from the total meanings of its elements. Vividness is scarce amenable to analysis at all, for to a certain extent the vividness of the complex as a whole is inversely proportional to the vividness of its elements. Local significance may become entirely lost in a complex, especially when the complex involves the coöperation of two or more senses, and it appears in these cases only by the analysis of the complex into its elements.

With regard to their analytic behaviour therefore, as well as their essentiality, the feeling-tone, meaning, vividness, and local significance characters differ radically from quality, intensity, duration, and extensity.

There is however one way in which quality differs fundamentally from intensity, duration, and extensity, and that is in its method of variation. The variations in these last three are continuous from zero to the highest possible value, without any points of special value, $i . e$, determining points, in the continuum; while the variations of quality pass through definite special or determining points with transition regions between. This difference is formulated in another way in the statement that variation in quality is variation in kind, while variations in intensity, duration, and extensity are not in themselves variations in kind.

As regards the physiological concomitants of at least five of these characters of simple sense objects, we can speak with confidence. Quality is essentially correlated with the kind 
of end organ stimulated (or perhaps with the kind of process which is aroused in the end organ and brain cell). Intensity is correlated with the intensity of the process aroused. Duration is identical with the duration of the process, presumably in the central cell. Local significance is the correlate of the particular end organs stimulated; we may suppose each end organ to have its particular local sign. Extensity has in this respect its full title proven, since it is obviously the correspondent of the num. ber of contiguous end organs stimulated.

The physiological conditions of the other characters - feeling-tone, meaning and vividness - are much more complex, and hence, though we assume that they are perfectly definite, are not readily assignable.

This scheme, of four essential characters, and four which may be called accidental, is satisfactory until we come to the consideration of sound sensations, at which point it seems at first unsatisfactory. Have we any character with which pitch can be identified? Duration, intensity, vividness, feeling-tone and meaning are of course excluded from the possibility, since they all apply to sounds over and above pitch; so quality, extensity and local significance are left to be considered. Quality, however, is soon put out of the running, since we have shown that in the other senses it varies through various determining points which are few in number for each sense; whereas pitch varies in a continuum without internal orientation, exactly as do intensity, duration, extensity and vividness. Local significance is the character which we should urge in this connection, if we held to the Helmholtz theory of audition, but since the present disposition is to look upon that theory as mechanically untenable, we should be obliged to look for further reasons for our choice, and such reasons, apart from the necessity of finding some character with which the identification may possibly be made, are not forthcoming. On the contrary, it seems impossible that pitch can rest upon local signs, since local signs do not in general vary between two extremes, but rather include a manifold of differences which do not admit of easy schematization. The same fact is expressed when we say that intensity, duration, extensity, and likewise pitch, admit of quantitative comparison, while local signs do not. 
Extensity, however, furnishes conditions which correspond in every particular to the properties of pitch. It varies continuously between extremes, admits of quantitative estimation more or less exactly, and moreover is directly connected with pitch in introspective analysis. The so-called ' high' notes are small. The 'low' notes are large or voluminous. Differences in pitch, in other words, are directly comparable to differences in planar or linear extent, and the physiological condition of difference in pitch accordingly is probably difference in number of nerve-endings stimulated. ${ }^{1}$

This theory of pitch is much more in accordance with the known facts of tone perception than is the Helmholtz theory. Let us consider first the discrimination of overtones. According to the local sign theory there ought to be very little difficulty in recognizing the octave when sounded with the fundamental, for the two components are dependent on the stimulation of two nerve endings or groups of nerve endings which must be relatively a considerable distance apart in the series and hence as easily discriminable as two points of light on the retina. On the extensity theory, however, the nerve endings which the higher note stimulates are all stimulated by the lower note; that is, the higher note is contained in the lower note both psychologically and physiologically, just as if a short streak of light were superposed on a long one; so that the discrimination where the notes harmonize (i.e., where there are no beats), and where the lower (or larger) note is not much less intense than the higher (or smaller), should be rather difficult, which is actually the case.

Moreover, if differences in pitch depended on differences in individual nerve endings stimulated, there would be no reason for expecting the lower note in a complex to dominate, $i . e$. , to give its pitch to the complex. But if the difference in pitch is really a difference in volume, we might well expect the larger to determine the size of the total complex, as really is the case when the lower note is not too weak.

${ }^{1}$ Subsequent to the construction of this theory on purely psychological grounds, I found that Ter Kuile had constructed a phyaiological theory with which it practically agrees. See Pfirger's Archiv, 1900, Vol. 79, pp. 146-157 and 484-509. 
In the third place, as regards a changing pitch, we have on either theory something analogous to a perception of motion; but on the local sign theory the analogous motion would be that of a point generating a line in the field of vision or of touch; while on the extensity theory the proper analogy would be a line increasing or decreasing in length; $i$. e., there is a certain part of the object which remains unchanged by the variation. This is a point on which introspection may differ in different cases, and on which it is hardly trustworthy on account of the sophistication of our auditory sense; in my own observation, however, the shrinking or expanding in linear extension in the field of vision or touch is a perfect analogue of the shifting pitch, and the moving point is not at all applicable.

Finally, the peculiarities of the complex sound we call ' noise, fit in perfectly with the proposed theory. The analytic characteristics of a 'noise' are: (I) excessive complexity, which is the essential feature, and may be the only one except for the beats to which it gives rise; (2) progressive variation in the intensity and pitch of the components, which is an accidental feature, but which adds greatly to the 'ncisiness'; and (3) indefiniteness in pitch of the complex determined by the first two characteristics.

The reason for this indefinitness of pitch becomes clear as soon as we consider the analogy to light sensations. Suppose we superpose many streaks of light of various lengths, making one end of each streak coincident with the corresponding ends of all the others. The result will be a streak of light relatively very intense at one end and fading off at the other, so that the length of the total illuminated area is indefinite. This is just what happens in the case of a noise; the superposition of the variety of tones makes the exact limits of the complex difficult of determination, although there is a general pitch distinguishable.

The designation of pitch as the form which extensity takes in auditory sensation seems to be the most satisfactory clearing up of the field of sensation characters. Not only does it dis pose of the vexed question of pitch, but it also helps to confirm the right of extensity to be considered an essential character of sensation, instead of an accidental character.

${ }^{2}$ The MS. of this article was received February 2, '05. - EpD. 